\title{
HARDY-POINCARÉ INEQUALITIES AND APPLICATIONS TO NONLINEAR DIFFUSIONS
}

\author{
A. BLANCHET, M. BONFORTE, J. DOLBEAULT, G. GRILLO, J.-L. VÁZQUEZ
}

\begin{abstract}
We systematically study weighted Poincaré type inequalities which are closely connected with Hardy type inequalities and establish the form of the optimal constants in some cases. Such inequalities are then used to relate entropy with entropy production and get intermediate asymptotics results for fast diffusion equations.
\end{abstract}

\section{Inégalités de Hardy-Poincaré et applications.}

RÉSumÉ. Nous étudions des inégalités de Poincaré qui sont étroitement reliées à des inégalités de type Hardy et établissons la forme des constantes optimales dans certains cas. De telles inégalités sont ensuite utilisées pour relier l'entropie avec la production d'entropie et obtenir des résultats d'asymptotiques intermédiaires pour les équations à diffusion rapide.

\section{VERSION FRANÇAISE ABRÉGÉE}

Dans cette note, nous nous intéressons à l'inégalité de Hardy-Poincaré

$$
\int_{\mathbb{R}^{d}} \frac{|v|^{2}}{1+|x|^{2}} d \mu_{\alpha} \leq \mathcal{C}_{\alpha, d} \int_{\mathbb{R}^{d}}|\nabla v|^{2} d \mu_{\alpha} \quad \text { avec } \quad d \mu_{\alpha}(x):=h_{\alpha}(x) d x, \quad h_{\alpha}(x):=\left(1+|x|^{2}\right)^{\alpha}
$$

pour $\alpha \in \mathbb{R} \backslash\left\{\alpha^{*}\right\}, \alpha^{*}=\alpha^{*}(d):=-(d-2) / 2, d \geq 3$. Si $\alpha<\alpha^{*}, d \mu_{\alpha-1}$ est bornée et on supposera alors $\int_{\mathbb{R}^{d}} v d \mu_{\alpha-1}=0$. Les inégalités de Hardy et Poincaré usuelles sont obtenues par changement d'échelle. Plus précisément, en utilisant l'estimation $\lim _{\varepsilon \rightarrow 0} \mathcal{C}_{-\varepsilon^{-2}, d} / \varepsilon^{2}=1 / 2$ et en passant à la limite $\varepsilon \rightarrow 0$, on obtient

$$
\int_{\mathbb{R}^{d}}|v|^{2} d \nu_{\infty} \leq \frac{1}{2} \int_{\mathbb{R}^{d}}|\nabla v|^{2} d \nu_{\infty} \quad \text { avec } \quad d \nu_{\infty}(x):=e^{-|x|^{2}} d x
$$

sous la condition $\int_{\mathbb{R}^{d}} v e^{-|x|^{2}} d x=0$. Par le changement d'échelle inverse, on obtient

$$
\int_{\mathbb{R}^{d}} \frac{|v|^{2}}{|x|^{2}} d \nu_{0, \alpha} \leq \kappa_{\alpha}^{-1} \int_{\mathbb{R}^{d}}|\nabla v|^{2} d \nu_{0, \alpha} \quad \text { avec } \quad d \nu_{0, \alpha}(x):=|x|^{2 \alpha} d x
$$

sous la condition $\bar{v}_{\alpha}:=\int_{\mathbb{R}^{d}} v d \nu_{0, \alpha}=0$ si $\alpha<\alpha^{*}$. Pour rendre finis les deux membres de l'inégalité, des précautions doivent être prises sur le comportement de $v$ en $x=0$. On supposera plus simplement que $\operatorname{supp}(v) \subset \mathbb{R}^{d} \backslash\{0\}$ et $\bar{v}_{\alpha}=0$. Une telle inégalité se démontre facilement avec $\kappa_{\alpha}=(d+2 \alpha-2)^{2} / 4$ en "développant le carré," $\left.\left.\int_{\mathbb{R}^{d}}\left|\nabla v+\left(\alpha-\alpha^{*}\right) x\right| x\right|^{-2} v\right|^{2}|x|^{2 \alpha} d x$, et en intégrant par parties. Notre principal résultat est le suivant.

Théorème 1. Supposons que $d \geq 3, \alpha \in \mathbb{R} \backslash\left\{\alpha^{*}\right\}$. L'inégalité (1) est vérifiée avec une constante $\mathcal{C}_{\alpha, d}>0$ (voir estimations ci-dessous), pour tout $v \in \mathcal{D}\left(\mathbb{R}^{d}\right)$, sous la condition additionnelle $\int_{\mathbb{R}^{d}} v d \mu_{\alpha-1}=0$ si $\alpha \in\left(-\infty, \alpha^{*}\right)$.

\begin{tabular}{|c|c|c|c|c|c|c|c|}
\hline$\alpha$ & $-\infty<\alpha \leq-d$ & $-d<\alpha<\alpha^{*}$ & $\alpha^{*}<\alpha \leq 1$ & $1 \leq \alpha \leq \bar{\alpha}(d)$ & $\bar{\alpha}(d) \leq \alpha \leq d$ & $d$ & $\alpha>d$ \\
\hline $\mathcal{C}_{\alpha, d}$ & $\frac{1}{2|\alpha|}$ & $\mathcal{C}_{\alpha, d} \geq \frac{4}{(d+2 \alpha-2)^{2}}$ & $\frac{4}{(d+2 \alpha-2)^{2}}$ & $\frac{4}{d(d+2 \alpha-2)}$ & $\frac{1}{\alpha(d+\alpha-2)}$ & $\frac{1}{2 d(d-1)}$ & $\frac{1}{d(d+\alpha-2)}$ \\
Optimalité & $?$ & $?$ & oui & $?$ & $?$ & oui & $?$ \\
\hline
\end{tabular}

Pour tout $\alpha>\alpha^{*}, \mathcal{C}_{\alpha, d} \geq 4 /(d+2 \alpha-2)^{2}=\kappa_{\alpha}^{-1}$ et $\bar{\alpha}(d)=\left(-d+\sqrt{5 d^{2}-16 d+16}+4\right) / 4 \in(1, d)$. D'autres estimations sont connues, voir [3] si $\alpha \in(-d,-d / 2)$ et [10] si $\alpha \in(-d,-(1+d / 2))$. Les inégalités dans des espaces de Sobolev à poids ont été abondamment étudiées: voir [8] et les références incluses. Plus spécifiquement pour (3), on renverra à $[7,14,12]$. L'une de nos estimations repose sur le lemme de Persson, [13], comme dans [5, 11], et utilise (3). Avec $\mathcal{H}_{R}:=\left\{v \in H^{1}\left(\mathbb{R}^{d}, d \mu_{\alpha}\right): \operatorname{supp}(v) \subset B(0, R)^{c}\right\}$, on montre que

$$
\mathcal{C}_{\alpha, d}^{-1} \in(0, \Lambda) \quad \text { avec } \quad \Lambda:=\inf \sigma_{\text {ess }}\left(-h_{1-\alpha} \nabla\left(h_{\alpha} \nabla \cdot\right)\right)=\lim _{R \rightarrow \infty} \inf _{v \in \mathcal{H}_{R}} \frac{\int_{\mathbb{R}^{d}}|\nabla v|^{2} d \mu_{\alpha}}{\int_{\mathbb{R}^{d}}|v|^{2} d \mu_{\alpha-1}} \geq \kappa_{\alpha}
$$


Le cas $\alpha \in(-\infty,-d)$ repose sur la linéarisation des inégalités d'interpolation optimales obtenues dans [9]. Si $\alpha \in$ $\left(\alpha^{*}, \bar{\alpha}(d)\right)$, on utilise une méthode qui repose sur une estimation de Cauchy-Schwarz astucieuse, voir [8], et fournit une constante optimale par comparaison avec (3). Le cas $\alpha \geq \bar{\alpha}(d)$ utilise l'inégalité de Sobolev correspondant à l'injection $H^{1}\left(\mathbb{R}^{d}\right) \hookrightarrow L^{2 d /(d-2)}\left(\mathbb{R}^{d}\right)$, ou plus précisément

$$
\frac{S}{S_{0}} \int_{\mathbb{R}^{d}} \frac{|u|^{2}}{\left(1+|x|^{2}\right)^{2}} d x \leq S\|u\|_{L^{\frac{2 d}{d-2}}\left(\mathbb{R}^{d}\right)}^{2} \leq \int_{\mathbb{R}^{d}}|\nabla u|^{2} d x
$$

avec égalité si $u=h_{-(d-2) / 2}$, appliquée à $u:=v h_{\alpha / 2}$.

La principale application du théorème 1 concerne l'équation de diffusion rapide

$$
u_{t}=\Delta u^{m}
$$

pour une donnée initiale positive ou nulle vérifiant $\int_{\mathbb{R}^{d}}\left(u_{0}-h_{\alpha}\right) d x=0, \alpha:=1 /(m-1)$ (des données initiales plus générales peuvent être obtenues par changement d'échelle). On supposera que $m \in(0,1)$ avec $m^{*}:=\max \{(d-$ $4) /(d-2), 0\}$, et on notera que pour $d \geq 4, \alpha\left(m^{*}\right)=\alpha^{*}(d)$. Par un changement d'échelle dépendant du temps, on ramène le problème à une équation de type Fokker-Planck, $v_{t}=\frac{1-m}{m} \Delta v^{m}+2 \operatorname{div}(x v)$ avec donnée initiale $v_{\mid t=0}=u_{0}$.

Corollaire 2. Soit, $d \geq 3, m \in(0,1), m \neq m^{*}, \alpha=1 /(m-1)$. Alors il existe $\varepsilon=\varepsilon(m, d) \in(0,1)$ et $K_{m, d}>0$ tels que pour tout $w \in L_{+}^{\infty}\left(\mathbb{R}^{d}\right)$ avec $\int_{\mathbb{R}^{d}}(w-1) h_{1} d \mu_{\alpha-1}=\int_{\mathbb{R}^{d}}(w-1) d \mu_{\alpha}=0,\|w-1\|_{L^{\infty}\left(\mathbb{R}^{d}\right)} \leq \varepsilon$, on ait

$$
\int_{\mathbb{R}^{d}} \frac{w^{m}-1-m(w-1)}{m-1} d \mu_{\alpha+1} \leq \mathcal{K}_{m, d} \int_{\mathbb{R}^{d}}\left|\nabla\left[\left(w^{m-1}-1\right) h_{1}\right]\right|^{2} w d \mu_{\alpha} .
$$

Lorsque $m \in[(d-1) / d, 1)$ et $m \in\left(m_{c},(d-1) / d\right), m_{c}:=(d-2) / d$, on renverra respectivement à [9] et [4]. Des résultats de convergence sans taux ont été obtenus récemment dans [6] pour $m<m_{c}$. Le second résultat principal de cette note, dont diverses généralisations, y compris pour $m \geq m_{c}$, seront données dans [1], s'énonce comme suit:

Théorème 3. Soit $d \geq 3, m \in\left(0, m_{c}\right), m \neq m^{*}$. Supposons que $u_{0}$ est une fonction positive ou nulle telle que $\left(u_{0}-h_{\alpha}\right) \in L^{1}\left(\mathbb{R}^{d}\right), \int_{\mathbb{R}^{d}}\left(u_{0}-h_{\alpha}\right) d x=0, \alpha=1 /(m-1)$. Supposons de plus que $\left(\sigma_{0}+|x|^{2}\right)^{1 /(m-1)} \leq u_{0}(x) \leq$ $\left(\sigma_{1}+|x|^{2}\right)^{1 /(m-1)}$ pour tout $x \in \mathbb{R}^{d}$, où $\sigma_{0} \in\left[1,(1-\varepsilon)^{1 /(m-1)}\right]$ et $\sigma_{1} \in\left[(1+\varepsilon)^{1 /(m-1)}, 1\right]$ avec $\varepsilon$ comme au Corollaire 2. Alors il existe une constante $\mathcal{C}$ telle que toute solution de (4) avec donnée initiale $u_{0}$ vérifie, pour tout $t \in(0, T(m))$,

$$
\left\|u(\cdot, t)-R^{-d}(t) h_{\alpha}\left(R^{-1}(t) \cdot\right)\right\|_{L^{2}\left(\mathbb{R}^{d}\right)} \leq \mathcal{C}\left(1-\frac{t}{T(m)}\right)^{\gamma}, \quad \gamma:=\left(\frac{d}{2}+\frac{m}{4 \mathcal{K}_{m, d}(1-m)}\right) \frac{1}{d\left(m_{c}-m\right)} .
$$

\section{INTRODUCTION AND MAIN RESUlT}

This note is devoted to the following inequalities

$$
\int_{\mathbb{R}^{d}} \frac{|v|^{2}}{1+|x|^{2}} d \mu_{\alpha} \leq \mathcal{C}_{\alpha, d} \int_{\mathbb{R}^{d}}|\nabla v|^{2} d \mu_{\alpha} \quad \text { with } \quad d \mu_{\alpha}(x):=h_{\alpha}(x) d x, \quad h_{\alpha}(x):=\left(1+|x|^{2}\right)^{\alpha}
$$

for $\alpha \in \mathbb{R} \backslash\left\{\alpha^{*}\right\}, \alpha^{*}=\alpha^{*}(d):=-(d-2) / 2, d \geq 3$. Notice that $\int_{\mathbb{R}^{d}} d \mu_{\alpha-1}$ is finite if $\alpha<\alpha^{*}$, and in such a case, we further assume that $\int_{\mathbb{R}^{d}} v d \mu_{\alpha-1}=0$. We call Inequality (1) a Hardy-Poincaré inequality for the following reasons.

Concerning Poincaré's inequality, notice that if we apply (1) with $\alpha=-1 / \varepsilon^{2}$ to $v_{\varepsilon}(x):=\varepsilon^{-d / 2} v(x / \varepsilon)$, we get

$$
\int_{\mathbb{R}^{d}}|v|^{2}\left(1+\varepsilon^{2}|x|^{2}\right)^{-1 / \varepsilon^{2}-1} d x \leq \mathcal{C}_{-1 / \varepsilon^{2}, d} / \varepsilon^{2} \int_{\mathbb{R}^{d}}|\nabla v|^{2}\left(1+\varepsilon^{2}|x|^{2}\right)^{-1 / \varepsilon^{2}} d x .
$$

Assuming that $\lim _{\varepsilon \rightarrow 0} \mathcal{C}_{-\varepsilon^{-2}, d} / \varepsilon^{2}=1 / 2$ (see Proposition 5 ) and passing to the limit as $\varepsilon \rightarrow 0$, we get

$$
\int_{\mathbb{R}^{d}}|v|^{2} d \nu_{\infty} \leq \frac{1}{2} \int_{\mathbb{R}^{d}}|\nabla v|^{2} d \nu_{\infty} \quad \text { with } \quad d \nu_{\infty}(x):=e^{-|x|^{2}} d x
$$

Such an inequality holds true only if $\int_{\mathbb{R}^{d}} v e^{-|x|^{2}} d x=0$, which is consistent with the requirement that (1) holds as $\alpha \rightarrow-\infty$ only under the condition $\int_{\mathbb{R}^{d}} v d \mu_{\alpha-1}=0$ (to fulfill such an inequality uniformly with respect to $\alpha$, some special conditions have to be assumed on $v$, for instance that $v(-x)=-v(x)$ for any $\left.x \in \mathbb{R}^{d}\right)$. 
As for Hardy's inequality, by applying (1) to $v_{1 / \varepsilon}(x):=\varepsilon^{d / 2} v(\varepsilon x)$ (with $v(-x)=-v(x)$ for any $x \in \mathbb{R}^{d}$ as above, if $\alpha<\alpha^{*}$ ), we get

$$
\int_{\mathbb{R}^{d}}|v|^{2}\left(\varepsilon^{2}+|x|^{2}\right)^{\alpha-1} d x \leq \mathcal{C}_{\alpha, d} \int_{\mathbb{R}^{d}}|\nabla v|^{2}\left(\varepsilon^{2}+|x|^{2}\right)^{\alpha} d x
$$

so that by passing to the limit $\varepsilon \rightarrow 0$, for a given $\alpha \in \mathbb{R}$, with $\kappa_{\alpha} \geq 1 / \mathcal{C}_{\alpha, d}$, we obtain

$$
\int_{\mathbb{R}^{d}} \frac{|v|^{2}}{|x|^{2}} d \nu_{0, \alpha} \leq \kappa_{\alpha}^{-1} \int_{\mathbb{R}^{d}}|\nabla v|^{2} d \nu_{0, \alpha} \quad \text { with } \quad d \nu_{0, \alpha}(x):=|x|^{2 \alpha} d x
$$

Such an inequality holds true only if $\bar{v}_{\alpha}:=\int_{\mathbb{R}^{d}} v d \nu_{0, \alpha}=0$ if $\alpha<\alpha^{*}$. Still both sides of the inequality might be infinite without further restrictions. With $u$ bounded in a neighborhood of $x=0$ and sufficiently decaying at infinity, the l.h.s. of the inequality is bounded if $\alpha>\alpha^{*}$. For $\alpha<\alpha^{*}$, if $v$ is continuous, then $v(0)=0$ has to be imposed: To avoid further discussions, we will simply require that $\operatorname{supp}(v) \subset \mathbb{R}^{d} \backslash\{0\}$ and $\bar{v}_{\alpha}=0$.

Notice that (3) is easy to establish by the "completing the square method" as follows. Let $v \in \mathcal{D}\left(\mathbb{R}^{d}\right)$ (with $\operatorname{supp}(v) \subset \mathbb{R}^{d} \backslash\{0\}$ if $\left.\alpha<\alpha^{*}\right)$.

$$
0 \leq \int_{\mathbb{R}^{d}}\left|\nabla v+\lambda \frac{x}{|x|^{2}} v\right|^{2}|x|^{2 \alpha} d x=\int_{\mathbb{R}^{d}}|\nabla v|^{2}|x|^{2 \alpha} d x+\left[\lambda^{2}-\lambda(d+2 \alpha-2)\right] \int_{\mathbb{R}^{d}} \frac{|v|^{2}}{|x|^{2}}|x|^{2 \alpha} d x .
$$

An optimization of the right hand side with respect to $\lambda$ gives $\lambda=\alpha-\alpha^{*}$, that is $\kappa_{\alpha}=(d+2 \alpha-2)^{2} / 4=\lambda^{2}$. Such an inequality is optimal, with optimal constant $\kappa_{\alpha}$, as follows by considering the test functions: 1) $v_{\varepsilon}(x)=$ $\min \left\{\varepsilon^{-\lambda},\left(|x|^{-\lambda}-\varepsilon^{\lambda}\right)_{+}\right\}$if $\left.\alpha>\alpha^{*}, 2\right) v_{\varepsilon}(x)=|x|^{1-\alpha-d / 2+\varepsilon}$ for $|x|<1$ and $v_{\varepsilon}(x)=(2-|x|)_{+}$for $|x| \geq 1$ if $\alpha<\alpha^{*}$, and letting $\varepsilon \rightarrow 0$ in both cases.

Theorem 1. Assume that $d \geq 3, \alpha \in \mathbb{R} \backslash\left\{\alpha^{*}\right\}$. Then (1) holds for some positive constant $\mathcal{C}_{\alpha, d}$, for any $v \in \mathcal{D}\left(\mathbb{R}^{d}\right)$, under the additional condition $\int_{\mathbb{R}^{d}} v d \mu_{\alpha-1}=0$ if $\alpha \in\left(-\infty, \alpha^{*}\right)$.

The proof will be split in several partial results and explicit estimates are summarized in Table 1. For simplicity, we consider only the case of the euclidean space $\mathbb{R}^{d}$, but some of our result can be extended to domains in $\mathbb{R}^{d}$ or to manifolds. The inequality applies to any function $v$ in the natural weighted Sobolev space obtained as the completion of $\mathcal{D}\left(\mathbb{R}^{d}\right)$ with respect to the norm $\|v\|_{\alpha}:=\left(\int_{\mathbb{R}^{d}}|\nabla v|^{2} d \mu_{\alpha}+\int_{\mathbb{R}^{d}}|v|^{2} d \mu_{\alpha+1}\right)^{1 / 2}$. One of our partial results relies as in $[5,11]$ on an abstract spectral argument involving Persson's characterization of the continuous spectrum of an operator, see [13], and exploits the information given by (3). Inequalities with weights have been studied by many authors, see for instance [8] and references therein. For a review on Hardy's inequality, we refer more specifically to [7]. Also see [14, 12]. We do not claim originality for Inequality (1) for all values of $\alpha$ since many partial results are scattered in the existing literature. Our goal is to give a unified and systematic presentation, with in mind the application to porous media $(\alpha>0)$ and fast diffusion $(\alpha<0)$ equations. An illustration with new results in the range $\alpha \in\left(-d / 2, \alpha^{*}\right)$ is given in last section. More details will be given in [1].

\section{Proof of the Main Result}

Proposition 2. Assume that $d \geq 3, \alpha>0$. Then (1) holds with $1 / \mathcal{C}_{\alpha, d}=(d-2+\alpha) \min \{\alpha, d\}$ for any $v \in \mathcal{D}\left(\mathbb{R}^{d}\right)$. The constant $\mathcal{C}_{\alpha, d}$ is optimal if $\alpha=d$.

Proof. Let $h_{\alpha}(x):=\left(1+|x|^{2}\right)^{\alpha}$. By Hölder's and Sobolev's inequalities, with $S_{0}:=\left\|h_{-d / 2}\right\|_{L^{2}\left(\mathbb{R}^{d}\right)}^{2}$, we get

$$
\frac{S}{S_{0}} \int_{\mathbb{R}^{d}} \frac{|u|^{2}}{\left(1+|x|^{2}\right)^{2}} d x \leq S\|u\|_{L^{\frac{2 d}{d-2}}\left(\mathbb{R}^{d}\right)}^{2} \leq \int_{\mathbb{R}^{d}}|\nabla u|^{2} d x,
$$

where the equality case is achieved by $h_{-(d-2) / 2}$ and $S$ is the best constant in Sobolev's inequality. Applied to $u:=v h_{\alpha / 2}$, this gives

$$
\frac{S}{S_{0}} \int_{\mathbb{R}^{d}}|v|^{2}\left(1+|x|^{2}\right)^{\alpha-2} d x \leq \int_{\mathbb{R}^{d}}\left[|\nabla v|^{2}-\alpha \frac{d+(d-2+\alpha)|x|^{2}}{\left(1+|x|^{2}\right)^{2}}|v|^{2}\right]\left(1+|x|^{2}\right)^{\alpha} d x .
$$

Define $I_{a}:=\int_{\mathbb{R}^{d}} h_{-a} d x$. To compute $S / S_{0}=(d-2)^{2}\left(I_{d-1}-I_{d}\right) / I_{d}$, we notice that an integration by parts gives $I_{d-1}-I_{d}=d I_{d-1} /(2(d-1))$, so that $S / S_{0}=d(d-2)$. Collecting the estimates, we obtain

$$
(d-2+\alpha) \int_{\mathbb{R}^{d}}|v|^{2}\left(1+|x|^{2}\right)^{\alpha-2}\left(d+\alpha|x|^{2}\right) d x \leq \int_{\mathbb{R}^{d}}|\nabla v|^{2}\left(1+|x|^{2}\right)^{\alpha} d x,
$$


which proves the estimate on $\mathcal{C}_{\alpha, d}$ for any $\alpha>0$. All above inequalities become equalities for $u=h_{-(d-2) / 2}$, if $\alpha=d$.

Proposition 3. Let $d \geq 3, \alpha \in\left(\alpha^{*}, \infty\right)$. Then (1) holds for any $v \in \mathcal{D}\left(\mathbb{R}^{d}\right)$ with $\mathcal{C}_{\alpha, d}:=4 /(d-2+2 \alpha)^{2}$ if $\alpha \in\left(\alpha^{*}, 1\right]$ and $\mathcal{C}_{\alpha, d}:=4 /[d(d-2+2 \alpha)]$ if $\alpha \geq 1$. The constant $\mathcal{C}_{\alpha, d}$ is optimal for any $\alpha \in\left(\alpha^{*}, 1\right]$.

Proof. We compute $\nabla h_{\alpha}=2 \alpha x h_{\alpha-1}$ and observe that $\Delta h_{\alpha}=2 \alpha h_{\alpha-2}\left[d+2\left(\alpha-\alpha^{*}\right)|x|^{2}\right]$ has a sign. An integration by parts and the Cauchy-Schwarz inequality shows that

$$
\left.\left|\int_{\mathbb{R}^{d}}\right| v\right|^{2} \Delta h_{\alpha} d x\left|\leq 2 \int_{\mathbb{R}^{d}}\right| v|| \nabla v|| \nabla h_{\alpha} \mid d x \leq 2\left(\int_{\mathbb{R}^{d}}|v|^{2}\left|\Delta h_{\alpha}\right| d x\right)^{1 / 2}\left(\int_{\mathbb{R}^{d}}|\nabla v|^{2}\left|\nabla h_{\alpha}\right|^{2}\left|\Delta h_{\alpha}\right|^{-1} d x\right)^{1 / 2} .
$$

As in [8], we remark that $\Delta h_{\alpha}$ has a constant sign and get the estimate

$$
\left.\left.\left|\int_{\mathbb{R}^{d}}\right| v\right|^{2} \Delta h_{\alpha} d x\left|=\int_{\mathbb{R}^{d}}\right| v\right|^{2}\left|\Delta h_{\alpha}\right| d x \leq 4 \int_{\mathbb{R}^{d}}|\nabla v|^{2}\left|\nabla h_{\alpha}\right|^{2}\left|\Delta h_{\alpha}\right|^{-1} d x .
$$

Weights can be estimated on both sides of the inequality:

$$
\begin{aligned}
& \left|\Delta h_{\alpha}\right|=2|\alpha|\left(1+|x|^{2}\right)^{\alpha-1} \frac{d+(d-2+2 \alpha)|x|^{2}}{1+|x|^{2}} \geq 2|\alpha| \min \{d,(d-2+2 \alpha)\} \frac{h_{\alpha}(x)}{1+|x|^{2}}, \\
& \frac{\left|\nabla h_{\alpha}\right|^{2}}{\left|\Delta h_{\alpha}\right|}=\frac{2|\alpha||x|^{2}}{d+(d-2+2 \alpha)|x|^{2}} h_{\alpha}(x) \leq \frac{2|\alpha|}{d-2+2 \alpha} h_{\alpha}(x),
\end{aligned}
$$

which proves the result for $\alpha \neq 0$. The case $\alpha=0$ is obtained by taking the limit $\alpha \rightarrow 0$ or $h_{0}(x):=\log \left(1+|x|^{2}\right)$.

From Section 1, we know that $\mathcal{C}_{\alpha, d}:=4 /(d-2+2 \alpha)^{2}=\kappa_{\alpha}^{-1}$ is optimal in (3) and that (3) holds as a consequence of (1) by a scaling argument, with same constant. This proves the optimality statement.

Proposition 4. Let $d \geq 3, \alpha \in\left(-\infty, \alpha^{*}\right)$. Then (1) holds for some $\mathcal{C}_{\alpha, d} \geq 4 /(d+2 \alpha-2)^{2}$, for any $v \in \mathcal{D}\left(\mathbb{R}^{d}\right)$ such that $\int_{\mathbb{R}^{d}} v d \mu_{\alpha-1}=0$.

Proof. On $L^{2}\left(\mathbb{R}^{d}, d \mu_{\alpha-1}\right)$, consider the closable quadratic form $v \mapsto \mathcal{Q}[v]:=\int_{\mathbb{R}^{d}}|\nabla v|^{2} d \mu_{\alpha}$ and denote by $-\mathcal{L}$ the unique non-negative self-adjoint operator on $L^{2}\left(\mathbb{R}^{d}, d \mu_{\alpha-1}\right)$ associated with the closure of $\mathcal{Q}$. By Persson's Theorem, see $[13,11]$, with $\mathcal{H}_{R}:=\left\{v \in H^{1}\left(\mathbb{R}^{d}, d \mu_{\alpha}\right): \operatorname{supp}(v) \subset B(0, R)^{c}\right\}$,

$$
\inf \sigma_{\text {ess }}(-\mathcal{L})=\lim _{R \rightarrow \infty} \inf _{v \in \mathcal{H}_{R}} \frac{\mathcal{Q}[v]}{\int_{\mathbb{R}^{d}}|v|^{2} d \mu_{\alpha-1}} \geq \kappa_{\alpha},
$$

where $\kappa_{\alpha}$ is the constant in (3). The lowest eigenvalue of $-\mathcal{L}$ is $\lambda_{1}=0$ and corresponding eigenfunctions are the constants. Since $\lambda_{1}$ is non-degenerate, $\mathcal{Q}[v] \geq \lambda_{2} \int_{\mathbb{R}^{d}}|v|^{2} d \mu_{\alpha-1}$ for some $\lambda_{2} \in\left(0, \kappa_{\alpha}\right]$, for any $v \in L^{2}\left(\mathbb{R}^{d}, d \mu_{\alpha-1}\right)$ such that $\int_{\mathbb{R}^{d}} v d \mu_{\alpha-1}=0$. This proves the result for $\mathcal{C}_{\alpha, d}^{-1}=\lambda_{2}-\lambda_{1} \in\left(0, \inf \sigma_{\text {ess }}(-\mathcal{L})\right]$.

Proposition 5. Let $d \geq 3, \alpha \in(-\infty,-d]$. Then (1) holds with $\mathcal{C}_{\alpha, d}^{-1} \geq 2|\alpha|$, for any $v \in \mathcal{D}\left(\mathbb{R}^{d}\right)$ such that $\int_{\mathbb{R}^{d}} v d \mu_{\alpha-1}=0$.

Proof. Let $F[u]:=\frac{1}{2}\left(\frac{m}{m-1}\right)^{2} \int_{\mathbb{R}^{d}}\left|\nabla u^{m-1}-\nabla u_{\infty}^{m-1}\right|^{2} u d x-\frac{1}{m-1} \int_{\mathbb{R}^{d}}\left[u^{m}-u_{\infty}^{m}-m u_{\infty}^{m-1}\left(u-u_{\infty}\right)\right] d x$ where $u_{\infty}(x):=$ $\left(\sigma+\frac{1-m}{2 m}|x|^{2}\right)^{1 /(m-1)}, \sigma>0$ and $m \in((d-1) / d, 1)$ is such that $m=1+1 / \alpha$. According to the Gagliardo-Nirenberg inequality, see [9], for any nonnegative function $u \in L^{1}\left(\mathbb{R}^{d}\right)$ such that $\int_{\mathbb{R}^{d}} u d x=\int_{\mathbb{R}^{d}} u_{\infty} d x, F[u] \geq F\left[u_{\infty}\right]=0$. With $\sigma=(1-m) /(2 m)$, a Taylor expansion at order two in $\varepsilon$ of $F\left[u_{\infty}\left(1+\varepsilon u_{\infty}^{1-m} v\right)\right]$ gives Inequality (1) with $\mathcal{C}_{\alpha, d}^{-1} \geq 2|\alpha|$.

\begin{tabular}{|c|c|c|c|c|c|c|c|}
\hline$\alpha$ & $-\infty<\alpha \leq-d$ & $-d<\alpha<\alpha^{*}$ & $\alpha^{*}<\alpha \leq 1$ & $1 \leq \alpha \leq \bar{\alpha}(d)$ & $\bar{\alpha}(d) \leq \alpha \leq d$ & $d$ & $\alpha>d$ \\
\hline $\mathcal{C}_{\alpha, d}$ & $\frac{1}{2|\alpha|}$ & $\mathcal{C}_{\alpha, d} \geq \frac{4+2)^{2}}{(d+2 \alpha-2)^{2}}$ & $\frac{4}{(d+2 \alpha-2)^{2}}$ & $\frac{4}{d(d+2 \alpha-2)}$ & $\frac{1}{\alpha(d+\alpha-2)}$ & $\frac{1}{2 d(d-1)}$ & $\frac{1}{d(d+\alpha-2)}$ \\
Optimality & $?$ & $?$ & yes & $?$ & $?$ & yes & $?$ \\
See Proposition. & 5 & 4 & 3 & 3 & 2 & 2 & 2 \\
\hline
\end{tabular}

TABLE 1. The value $\bar{\alpha}(d)=\left(-d+\sqrt{5 d^{2}-16 d+16}+4\right) / 4 \in(1, d)$, is determined by the condition $4 /(d(d+2 \alpha-2))=1 /(\alpha(d+\alpha-2))$. For any $\alpha>\alpha^{*}, d \geq 3$ and $\mathcal{C}_{\alpha, d} \geq 4 /(d+2 \alpha-2)^{2}=\kappa_{\alpha}^{-1}$, which is optimal for Inequality (3) if $\alpha \in(\alpha *, 1)$. In the range $\alpha<\alpha^{*}$, other estimates are known: see [3] for $\alpha \in(-d,-d / 2)$ and $[10]$ for $\alpha \in(-d,-(1+d / 2))$. 


\section{Applications to the fast Diffusion EQUATions}

For any $m \in(0,1)$ with $m \neq m^{*}=m^{*}(d):=\max \{(d-4) /(d-2), 0\}$, let $\alpha=\alpha(m):=1 /(m-1)$ and notice that for $d \geq 4, \alpha\left(m^{*}\right)=\alpha^{*}(d)$. We recall the notations $d \mu_{\alpha}(x):=h_{\alpha}(x) d x$ with $h_{\alpha}(x):=\left(1+|x|^{2}\right)^{\alpha}$.

Corollary 6. Let $d \geq 3, m \in(0,1), m \neq m^{*}, \alpha=1 /(m-1)$. Then there exists $\varepsilon=\varepsilon(m, d) \in(0,1)$ and $K_{m, d}>0$ such that for any positive $w \in L^{\infty}\left(\mathbb{R}^{d}\right)$ with $\int_{\mathbb{R}^{d}}(w-1) h_{1} d \mu_{\alpha-1}=\int_{\mathbb{R}^{d}}(w-1) d \mu_{\alpha}=0,\|w-1\|_{L^{\infty}\left(\mathbb{R}^{d}\right)} \leq \varepsilon$,

$$
\mathrm{E}:=\int_{\mathbb{R}^{d}} \frac{w^{m}-1-m(w-1)}{m-1} d \mu_{\alpha+1} \leq \mathcal{K}_{m, d} \mid \quad \text { with } \mathbf{I}:=\int_{\mathbb{R}^{d}}\left|\nabla\left[\left(w^{m-1}-1\right) h_{1}\right]\right|^{2} w d \mu_{\alpha} .
$$

Proof. Let $\kappa_{0}(\varepsilon):=\max _{s \in[1-\varepsilon, 1+\varepsilon]} \frac{s^{m}-1-m(s-1)}{(m-1)(s-1)^{2}}$. On the one hand, $\mathrm{E}(t) \leq \kappa_{0}(\varepsilon) \int_{\mathbb{R}^{d}}|w-1|^{2} d \mu_{\alpha+1}$. On the other hand, using Inequality (1), we get $\int_{\mathbb{R}^{d}}|w-1|^{2} d \mu_{\alpha+1}=\int_{\mathbb{R}^{d}}\left|(w-1) h_{1}\right|^{2} d \mu_{\alpha-1} \leq \mathcal{C}_{\alpha, d} \int_{\mathbb{R}^{d}}\left|\nabla\left((w-1) h_{1}\right)\right|^{2} d \mu_{\alpha}$. Expanding the square and integrating by parts, we obtain, for some function $f$ to be fixed later,

$$
\int_{\mathbb{R}^{d}}\left|\nabla\left(f(w) h_{1}\right)\right|^{2} d \mu_{\alpha}=\int_{\mathbb{R}^{d}}\left|f^{\prime}(w)\right|^{2}|\nabla w|^{2} d \mu_{\alpha+2}+4|\alpha| \int_{\mathbb{R}^{d}}|x|^{2}|f(w)|^{2} d \mu_{\alpha}-2 d \int_{\mathbb{R}^{d}}|f(w)|^{2} d \mu_{\alpha+1} .
$$

Let $f_{m}(s):=s^{m-1}-1, \kappa_{1}(\varepsilon):=\max _{s \in[1-\varepsilon, 1+\varepsilon]}\left|\frac{f_{2}^{\prime}(s)}{f_{m}^{\prime}(s)}\right|^{2}$ and $\kappa_{2}(\varepsilon):=\min _{s \in[1-\varepsilon, 1+\varepsilon]}\left|\frac{f_{2}(s)}{f_{m}(s)}\right|^{2}$. With these notations,

$$
\left|f_{2}^{\prime}(w)\right|^{2} \leq \kappa_{1}(\varepsilon)\left|f_{m}^{\prime}(w)\right|^{2}, \quad\left|f_{2}(w)\right|^{2} \leq \kappa_{1}(\varepsilon)\left|f_{m}(w)\right|^{2} \quad \text { and } \quad\left|f_{m}(w)\right|^{2} \leq\left(\kappa_{2}(\varepsilon)\right)^{-1}\left|f_{2}(w)\right|^{2},
$$

and $\kappa_{1}(\varepsilon) \geq \kappa_{2}(\varepsilon)$. Hence, using successively $f=f_{2}$ and $f=f_{m}$, we get

$$
\int_{\mathbb{R}^{d}}\left|\nabla\left(f_{2}(w) h_{1}\right)\right|^{2} d \mu_{\alpha} \leq \kappa_{1}(\varepsilon) \int_{\mathbb{R}^{d}}\left|\nabla\left(f_{m}(w) h_{1}\right)\right|^{2} d \mu_{\alpha}+2 d\left[\frac{\kappa_{1}(\varepsilon)}{\kappa_{2}(\varepsilon)}-1\right] \int_{\mathbb{R}^{d}}\left|f_{2}(w)\right|^{2} d \mu_{\alpha+1} .
$$

For $\varepsilon$ small enough, $2 d\left[\kappa_{1}(\varepsilon) / \kappa_{2}(\varepsilon)-1\right]<1 / \mathcal{C}_{\alpha, d}$ and the result holds with

$$
\mathcal{K}_{m, d}=\frac{\kappa_{0}(\varepsilon) \kappa_{1}(\varepsilon) \kappa_{2}(\varepsilon) \mathcal{C}_{\alpha, d}}{\kappa_{2}(\varepsilon)+2 d\left[\kappa_{2}(\varepsilon)-\kappa_{1}(\varepsilon)\right] \mathcal{C}_{\alpha, d}} \frac{1}{1-\varepsilon}
$$

The inequality in Corollary 6 is motivated by the study of the asymptotic behavior of the solutions to the fast diffusion equation

$$
u_{t}=\Delta u^{m}
$$

for some nonnegative initial datum $u_{0}$. For simplicity, we shall assume that $\int_{\mathbb{R}^{d}}\left(u_{0}-h_{\alpha}\right) d x=0, \alpha=1 /(m-1)$. The general case can be deduced using an appropriate scaling. Let $m_{c}:=(d-2) / d, d \geq 3$. Two different regimes have to be distinguished. If $m \in\left(m_{c}, 1\right)$, solutions are well defined in $C^{0}\left(\mathbb{R}^{+}, L^{1}\left(\mathbb{R}^{d}\right)\right)$ and one is interested in understanding the behavior as $t \rightarrow \infty$. If $m \in\left(0, m_{c}\right)$ with $m \neq m^{*}$, at least some solutions vanish in a finite time $T=T(m)$, and one is interested in the asymptotic behavior as $t \rightarrow T$. For this purpose, we perform the following time-dependent rescaling

$$
u(t, x)=\frac{1}{R^{d}(t)} v\left(\tau(t), \frac{x}{R(t)}\right), \quad \tau(t)=\frac{1}{2} \log R(t), \quad R(t)=\left(1-\frac{t}{T(m)}\right)^{\frac{1}{d\left(m-m_{c}\right)}}, \quad T(m)=\frac{1-m}{2 d m\left(m_{c}-m\right)}
$$

with $T(m)<0$ and $t \in(0, \infty)$ if $m>m_{c}$, and $T(m)>0$ and $t \in(0, T(m))$ if $m<m_{c}$. The equation for $v$ is now of Fokker-Planck type

$$
v_{t}=\frac{1-m}{m} \Delta v^{m}+2 \operatorname{div}(x v)
$$

with initial data $v_{\mid t=0}=u_{0}$. This can be rewritten as $v_{t}+\operatorname{div}\left[v\left(\nabla\left(v^{m-1}\right)-2 x\right)\right]=0$. Let $w:=v / h_{\alpha}, \alpha=$ $1 /(m-1)$, and define the entropy and entropy production respectively by $\mathrm{E}(t):=\int_{\mathbb{R}^{d}} \frac{w^{m}-1-m(w-1)}{m-1} d \mu_{\alpha+1}$ and $\mathbf{I}(t):=\int_{\mathbb{R}^{d}}\left|\nabla\left[\left(w^{m}-1\right)\left(1+|x|^{2}\right)\right]\right|^{2} w d \mu_{\alpha}$. A simple computation and Corollary 6 show that $\frac{d}{d t} \mathrm{E}=\frac{m}{m-1} \mathrm{I}$, which is the key observation, while $\int_{\mathbb{R}^{d}}(w-1) d \mu_{\alpha}=\int_{\mathbb{R}^{d}}\left(v-h_{\alpha}\right)=0$ accounts for mass conservation.

Theorem 7. Let $d \geq 3, m \in\left(0, m_{c}\right), m \neq m^{*}$, and assume that $u_{0}$ is a nonnegative function such that $\left(u_{0}-h_{\alpha}\right) \in$ $L^{1}\left(\mathbb{R}^{d}\right), \int_{\mathbb{R}^{d}}\left(u_{0}-h_{\alpha}\right) d x=0, \alpha=1 /(m-1)$. Assume moreover that $\left(\sigma_{0}+|x|^{2}\right)^{1 /(m-1)} \leq u_{0}(x) \leq\left(\sigma_{1}+|x|^{2}\right)^{1 /(m-1)}$ 
for a.e. $x \in \mathbb{R}^{d}$, where $\sigma_{0} \in\left[1,(1-\varepsilon)^{1 /(m-1)}\right]$ and $\sigma_{1} \in\left[(1+\varepsilon)^{1 /(m-1)}, 1\right]$ with $\varepsilon$ as in Corollary 6 . Then there exists a constant $\mathcal{C}$ such that any solution of (4) with initial datum $u_{0}$ satisfies, for any $t \in(0, T(m))$,

$$
\left\|u(\cdot, t)-R^{-d}(t) h_{\alpha}\left(R^{-1}(t) \cdot\right)\right\|_{L^{2}\left(\mathbb{R}^{d}\right)} \leq \mathcal{C}\left(1-\frac{t}{T(m)}\right)^{\gamma}, \quad \gamma:=\left(\frac{d}{2}+\frac{m}{4 \mathcal{K}_{m, d}(1-m)}\right) \frac{1}{d\left(m_{c}-m\right)} .
$$

No smallness assumptions are required on $\sigma_{0}-1>0$ and $1-\sigma_{1}>0$ if we further assume that $\left\|u_{0} / h_{\alpha}-1\right\|_{L^{\infty}\left(\mathbb{R}^{d}\right)}<\varepsilon$. In the rescaled variables, we also obtain a moment estimate, that is a control on $\left\|v(\cdot, t)-h_{\alpha}\right\|_{L^{2}\left(\mathbb{R}^{d}, d \mu_{1-\alpha}\right)}$. Generalizations to other $L^{p}$-norms, $p \in[1,+\infty]$, will be given in [1]. Our results also apply to $m \in\left(m_{c}, 1\right)$ under the weaker assumptions $u_{0} \in L_{+}^{1}\left(\mathbb{R}^{d}\right)$ and $\limsup _{R \rightarrow \infty} \sup _{|x|>R}\left(|x|^{-2 /(m-1)} u_{0}(x)\right)<\infty$, since the assumptions of Theorem 7 are then satisfied for $t$ large enough, see [2] for details. For $m \in[(d-1) / d, 1)$ and $m \in\left(m_{c},(d-1) / d\right)$ with $m_{c}:=(d-2) / d$, we refer to [9] and [4] respectively. Convergence results without rate have recently been obtained in [6] for $m<m_{c}$. The case $m=m_{c}$ can be dealt with using the time-dependent rescaling given by $\tau(t)=t, R(t)=e^{t}$, while $m=m^{*}$ is out of reach with our methods. The assumption $\left(\sigma_{0}+|x|^{2}\right)^{1 /(m-1)} \leq u_{0}(x) \leq\left(\sigma_{1}+|x|^{2}\right)^{1 /(m-1)}$ is not a technical assumption, since a compactly supported initial datum has completely different asymptotics, see [15].

Sketch of the proof of Theorem 7. First of all, we observe that $\mathrm{E}(t)$ converges to 0 as $t \rightarrow \infty$. Using Corollary 6 , we obtain that $\int_{\mathbb{R}^{d}}|w-1|^{2} d \mu_{\alpha+1}=\int_{\mathbb{R}^{d}}\left|v-h_{\alpha}\right|^{2} d \mu_{1-\alpha}$ is controlled by $\mathrm{E}(t) \leq \mathrm{E}(0) \exp \left(-m \mathcal{K}_{m, d}^{-1} t /(1-m)\right)$. The result follows by noting that $\int_{\mathbb{R}^{d}}\left|v-h_{\alpha}\right|^{2} d x \leq \int_{\mathbb{R}^{d}}\left|v-h_{\alpha}\right|^{2} d \mu_{1-\alpha}$ and by undoing the change of variables.

(c) 2006 by the authors. This paper may be reproduced, in its entirety, for non-commercial purposes.

\section{REFERENCES}

[1] A. Blanchet, M. Bonforte, J. Dolbeault, G. Grillo, and J. L. Vazquez, Asymptotics of the fast diffusion equation in the very fast diffusion range. In preparation.

[2] M. Bonforte And J. L. VAzquez, Global positivity estimates and Harnack inequalities for the fast diffusion equation, J. Funct. Anal., 240 (2006), pp. 399-428.

[3] J. A. Carrillo, C. Lederman, P. A. Markowich, And G. Toscani, Poincaré inequalities for linearizations of very fast diffusion equations, Nonlinearity, 15 (2002), pp. 565-580.

[4] J. A. Carrillo and J. L. VÁzquez, Fine asymptotics for fast diffusion equations, Comm. Partial Differential Equations, 28 (2003), pp. 1023-1056.

[5] F. Cipriani, Sobolev-Orlicz imbeddings, weak compactness, and spectrum, J. Funct. Anal., 177 (2000), pp. 89-106.

[6] P. Daskalopoulos And N. Sesum, On the extinction profile of solutions to fast-diffusion, 2006.

[7] E. B. Davies, A review of Hardy inequalities, in The Maz'ya anniversary collection, Vol. 2 (Rostock, 1998), vol. 110 of Oper. Theory Adv. Appl., Birkhäuser, Basel, 1999, pp. 55-67.

[8] E. B. Davies And A. M. Hinz, Explicit constants for Rellich inequalities in $L_{p}(\Omega)$, Math. Z., 227 (1998), pp. 511-523.

[9] M. Del Pino and J. Dolbeault, Best constants for Gagliardo-Nirenberg inequalities and applications to nonlinear diffusions, J. Math. Pures Appl. (9), 81 (2002), pp. 847-875.

[10] J. Denzler And R. J. MCCann, Fast diffusion to self-similarity: complete spectrum, long-time asymptotics, and numerology, Arch. Ration. Mech. Anal., 175 (2005), pp. 301-342.

[11] G. Grillo, On Persson's theorem in local Dirichlet spaces, Z. Anal. Anwendungen, 17 (1998), pp. 329-338.

[12] B. Muckenhoupt, Hardy's inequality with weights, Studia Math., 44 (1972), pp. 31-38. Collection of articles honoring the completion by Antoni Zygmund of 50 years of scientific activity, I.

[13] A. Persson, Bounds for the discrete part of the spectrum of a semi-bounded Schrödinger operator, Math. Scand., 8 (1960), pp. 143-153.

[14] J. Piepenbrink, Integral inequalities and theorems of Liouville type, J. Math. Anal. Appl., 26 (1969), pp. $630-639$.

[15] J. L. VÁzquez, Smoothing and decay estimates for nonlinear diffusion equations, vol. 33 of Oxford Lecture Notes in Maths. and its Applications, Oxford Univ. Press, 2006.

A.B., M.B., J.D.: Ceremade, Université Paris Dauphine, Place de Lattre de Tassigny, 75775 Paris Cedex 16 , France E-mail address: blanchet, bonforte, dolbeaul@ceremade.dauphine.fr

G.G.: Dipartimento di Matematica, Politecnico di Torino, corso Duca degli Abruzzi 24, 10129 Torino, Italy E-mail address: gabriele.grillo@polito.it

J.L.V.: Departamento de Matemáticas, Universidad Autónoma de Madrid, Campus de Cantoblanco, 28049 Madrid, Spain E-mail address: juanluis.vazquez@uam.es 